Further developments will include extending the programme for the duration of the placement to enable us to cover an enhanced range of presentations and to consolidate core skills.

\section{The challenges of delivering addictions psychiatry teaching in the COVID era}

\author{
Rebecca Hammersley* and Amy Martin \\ Royal Edinburgh Hospital \\ ${ }^{*}$ Corresponding author.
}

doi: 10.1192/bjo.2021.395

Aims. During the pandemic, addiction psychiatry moved from face-to-face lectures (delivered by Addictions Psychiatrists) to bitesize pre-recorded lectures (delivered by clinical teaching fellows) alongside interactive tutorials (delivered by Addictions Psychiatrists). The Addictions Team developed an online tutorial (delivered via Blackboard Collaborate) containing a combination of information slides, case studies, interactive quizzes, and short videos. These were delivered 'live' to small groups of students in up to four simultaneous virtual classrooms on a 6-weekly rolling basis. We aimed to assess student and tutor feedback regarding the move to interactive online tutorials in addiction psychiatry.

Method. Two questionnaires sought feedback from students and tutors, focussing on the change from face-to-face to virtual teaching during a 20 -week period.

Result. 21 (of 161) students completed the questionnaire.

- $100 \%$ 'strongly agreed' or 'agreed' that the content of the tutorial was relevant to learning outcomes.

- $52 \%$ felt 'somewhat comfortable' unmuting their microphones to contribute verbally, contrasting to $24 \%$ feeling 'not very' or 'extremely uncomfortable'. In practice, only $30 \%$ of students contributed verbally.

- $57 \%$ felt most comfortable contributing via the written 'chat' function (rather than audio or camera).

- $65 \%$ felt either 'somewhat' or 'very comfortable' turning their cameras on, but only 1 student turned their camera on across all tutorials.

- $48 \%$ felt the tutorial was more accessible virtually than face-to-face; $5 \%$ considered it less so.

- When COVID restrictions are lifted, $14 \%$ would prefer the tutorial to remain virtual, 53\% would rather it returned face-to-face, and $33 \%$ had no preference.

7 (of 7) tutors completed the questionnaire.

- $100 \%$ felt that students having their camera on would make their experience of delivering teaching 'much better' or 'better'.

- $71 \%$ of tutors felt that students contributed 'slightly' or 'significantly' less in the virtual classroom.

- Only $29 \%$ of tutors found the experience of virtual teaching 'very' or 'somewhat' enjoyable, contrasting 43\% finding it 'somewhat' or 'very' unenjoyable.

- Several white space tutor comments suggested the lack of audiovisual engagement made teaching less rewarding, whilst also preventing them from adapting content, pace, and teaching style to suit the group's needs. Tutors felt that the ease of delivering teaching from any location was beneficial.

Conclusion. Virtual teaching has become embedded in medical education and will likely remain so post-pandemic. For it to be an effective and enjoyable experience, for both students and teachers, there needs to be adaptation of content, technology, etiquette and culture.

\section{Resilience-culture of support}

Nazish Hashmi ${ }^{1 \star}$ and Sunitha Muniyappl ${ }^{2}$

${ }^{1}$ Leeds and York NHS Partnership foundation trust and ${ }^{2}$ Bradford

NHS foundation trust

${ }^{\star}$ Corresponding author.

doi: 10.1192/bjo.2021.396

Aims. Better-informed trainees will have increased confidence and improved resilience which will have a positive impact on the workforce. To promote and celebrate diversity in psychiatric workforce it is imperative to acknowledge the above and provide adequate support to IMGs across UK.

Background. Nearly two fifth of licensed doctors in NHS are from black and ethnic minorities. Studies have shown that International Medical Graduates (IMGs) are particularly prone to certain difficulties compared to UK graduates. IMGs are more likely to be subject to investigations by General Medical Council for concerns over clinical skills and knowledge, communication skills, lack of awareness of the laws and code of practice. This has been highlighted by GMC as well as Royal College of Psychiatrists. To promote and celebrate diversity in psychiatric workforce it is imperative to acknowledge this and provide adequate support to IMGs across UK.

Method. An additional rotation wide induction programme was started for IMGs in August 2018 in West Yorkshire. This has continued on a 6 monthly basis for all new starters and last one was held on 21st of August 2019. Teaching included information about Good Medical Practice, confidentiality issues, principles of consent, information about living skills and practical teaching on phlebotomy and requesting investigations.

Result. The doctors who attended these sessions found it to be very helpful and some suggested it to be a full day programme. According to the feedback collected there was a definite improvement in understanding noted by IMGs in most areas covered. This induction was also acknowledged in the School of Psychiatry conference in October 2019.

Conclusion. Considering the increasing numbers of International medical graduates it will be beneficial to arrange similar events at local level for easier accessibility. In line with RCPsych and GMC guidelines all trusts should be encouraged to offer IMG induction sessions locally.

More than just the mental health act foundation-specific teaching to inspire psychiatrists

Robert Heminway*, Lauren Fitzmaurice, Hamid Alhaj and Edward Fearnley

Sheffield Health and Social Care NHS Foundation Trust ${ }^{\star}$ Corresponding author.

doi: $10.1192 /$ bjo.2021.397

Aims. This project aimed to further develop a teaching programme for Foundation Doctors attached to a psychiatry rotation. The purpose was threefold - to educate foundation doctors about important psychiatric topics; to encourage them to think about wider impacts of psychiatry; and to inspire them to consider psychiatric training in the longer term.

Background. The Royal College of Psychiatrists' mission statement includes actively promoting psychiatry as a career and 